\title{
Topological formation of Mo-Ni based hollow structure as a highly efficient electrocatalyst for hydrogen evolution reaction in alkaline solution
}

Yuxue Zhou, Min Luo, Wei Zhang, Zhenxin Zhang, Xiangdong Meng, Xiaoshuang

Shen, Hongfei Liu, Min Zhou*, Xianghua Zeng*

College of Physical Science and Technology, Institute of Optoelectronic

Technology, Yangzhou University, Yangzhou 225002, China. E-mail:

minzhou@yzu.edu.cn, xhzeng@yzu.edu.cn. 


\section{Supporting Information}

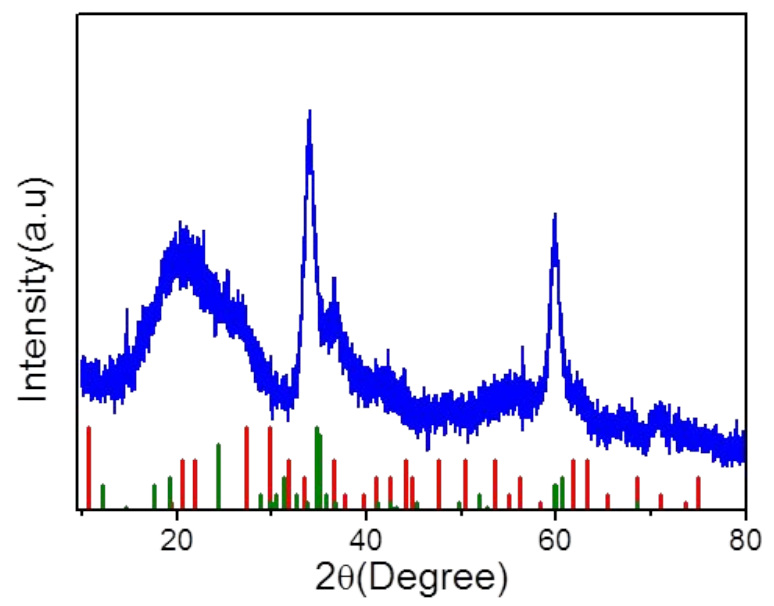

Figure S1.XRD pattern of MoNi-HS precursor. Red line: standard pattern of $\mathrm{NiMoO}_{4} \cdot \mathrm{xH}_{2} \mathrm{O}$ (JCPDS, No.130128), Green line: standard pattern of $\mathrm{Ni}_{2}\left(\mathrm{CO}_{3}\right)(\mathrm{OH})_{2}$ (JCPDS, No. 350501).

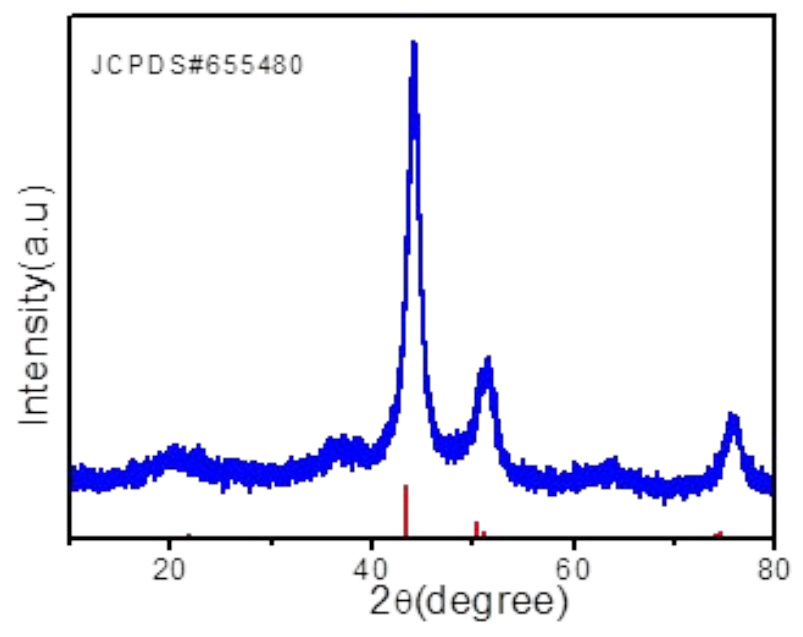

Figure S2.XRD pattern of MoNi-HS sample. 


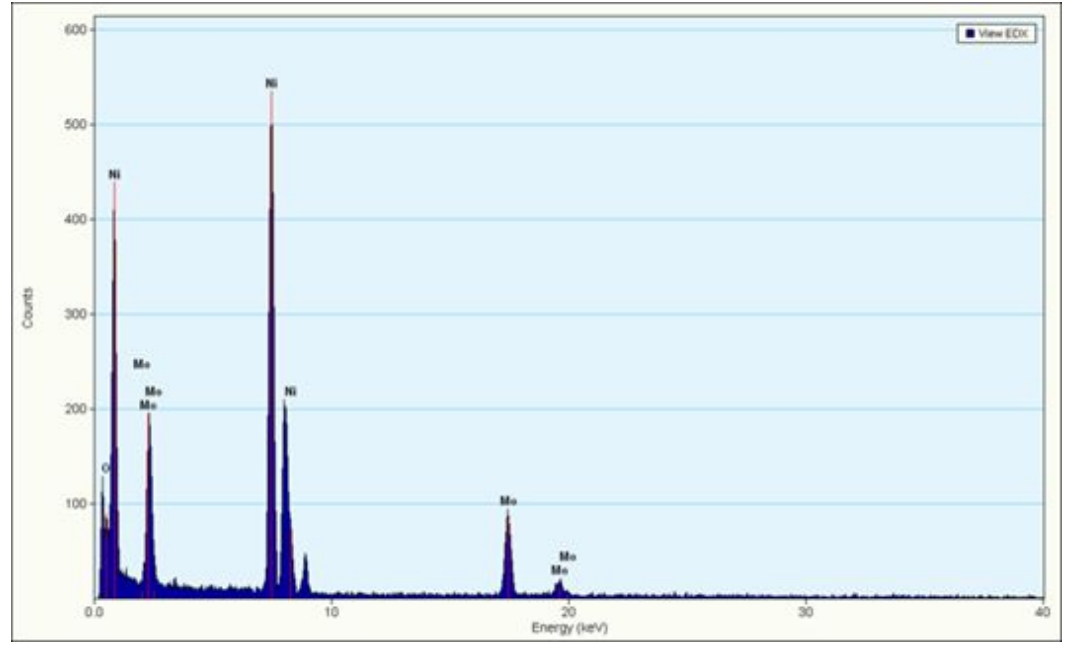

Figure S3. The corresponding energy dispersive X-ray (EDX) spectrum of MoNi-HS.

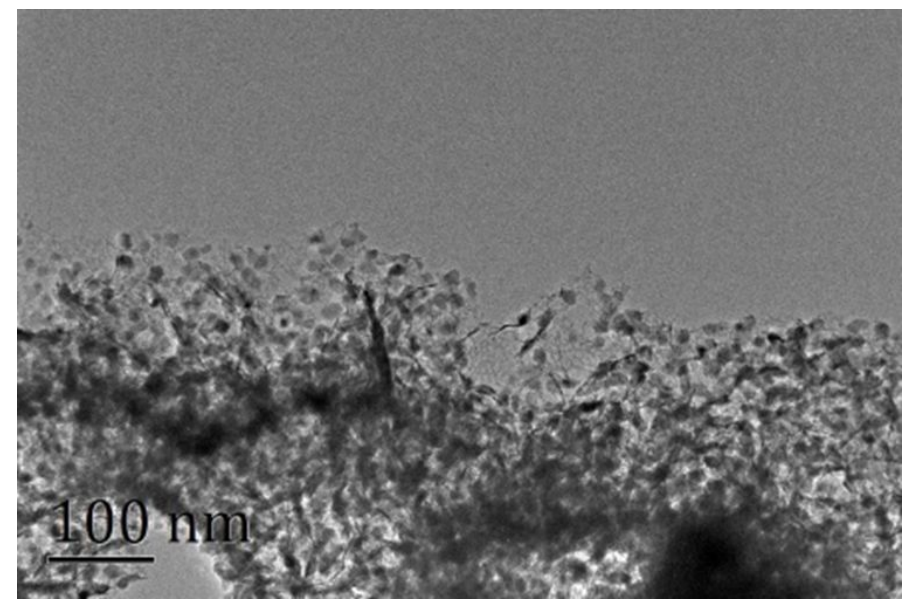

Figure S4. TEM image of MoNi-HS sample. 


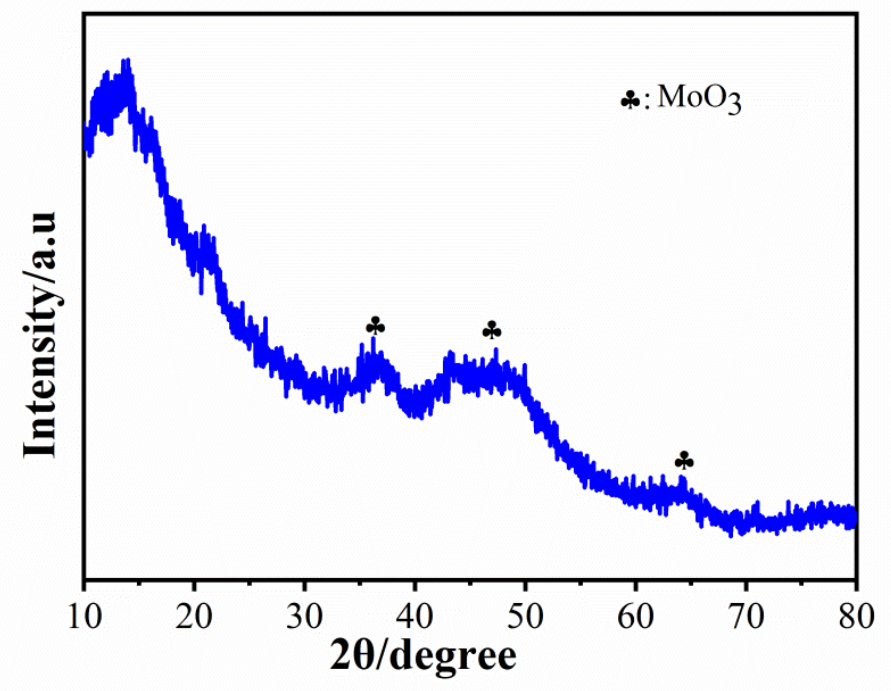

Figure S5. XRD pattern of remaining product from MoNi-HS after $0.1 \mathrm{M} \mathrm{H}_{2} \mathrm{SO}_{4}$ solution washing.
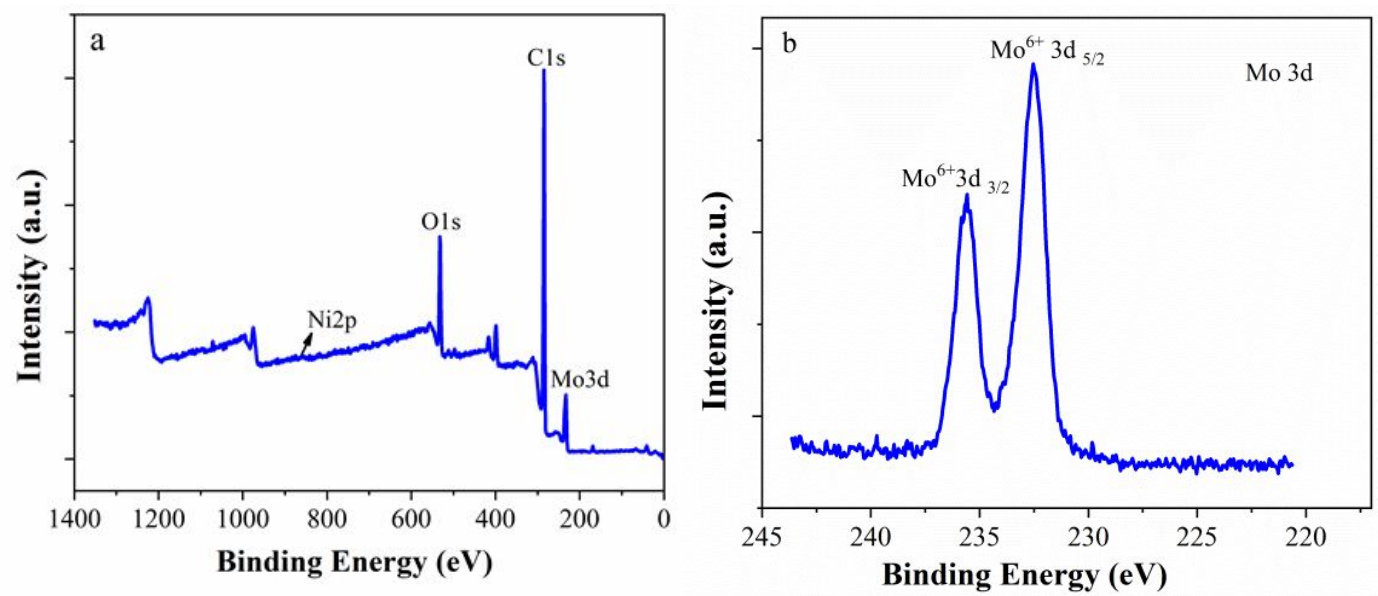

Figure S6.XPS spectra of remaining product from MoNi-HS after $0.1 \mathrm{M} \mathrm{H}_{2} \mathrm{SO}_{4}$ solution washing (a) full survey and (b) Mo 3d. 


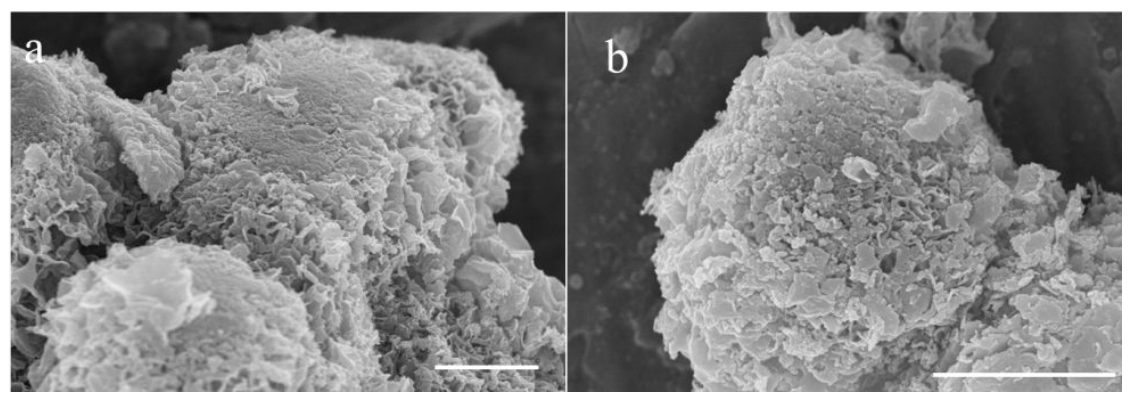

Figure S7. (a,b)SEM images of MoNi-HS-A. Scale bars (a,b)2 $\mu \mathrm{m}$.
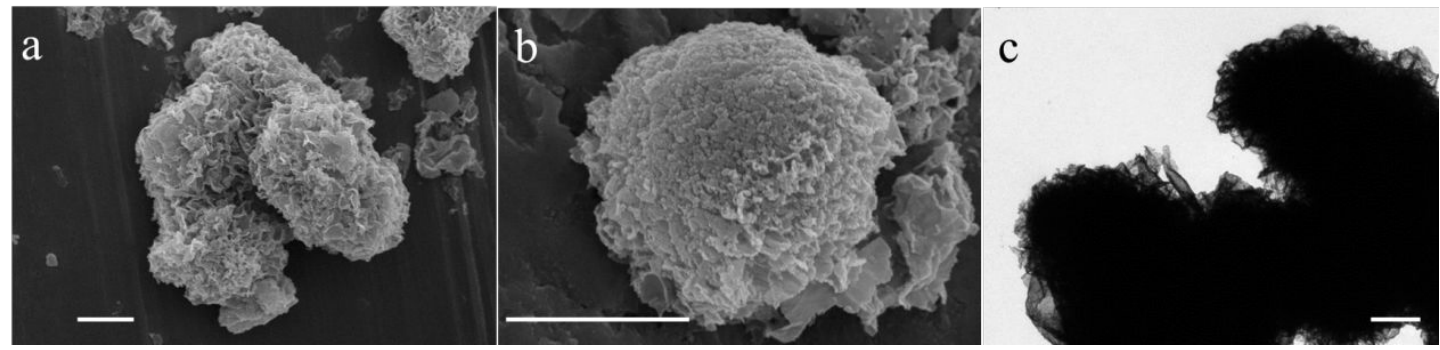

Figure S8. (a, b)SEM and (c) TEM image of MoNi-HS-A precursor. Scale bars (a, b) $2 \mu \mathrm{m}$ (c) $1 \mu \mathrm{m}$.

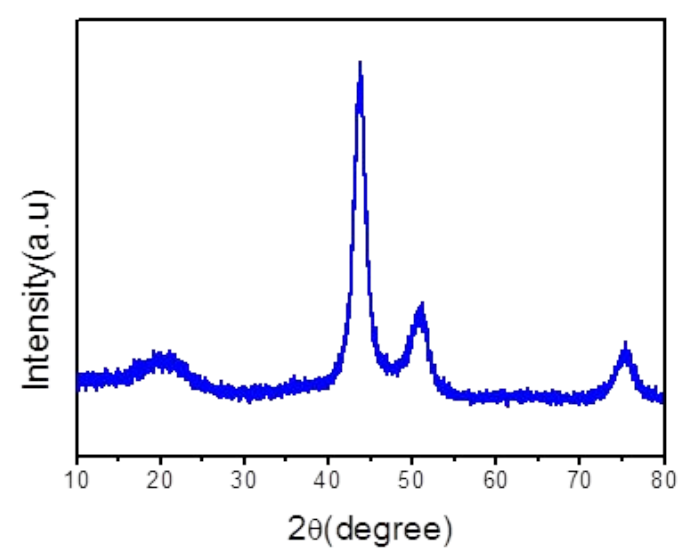

Figure S9. XRD pattern of MoNi-HS-A. 


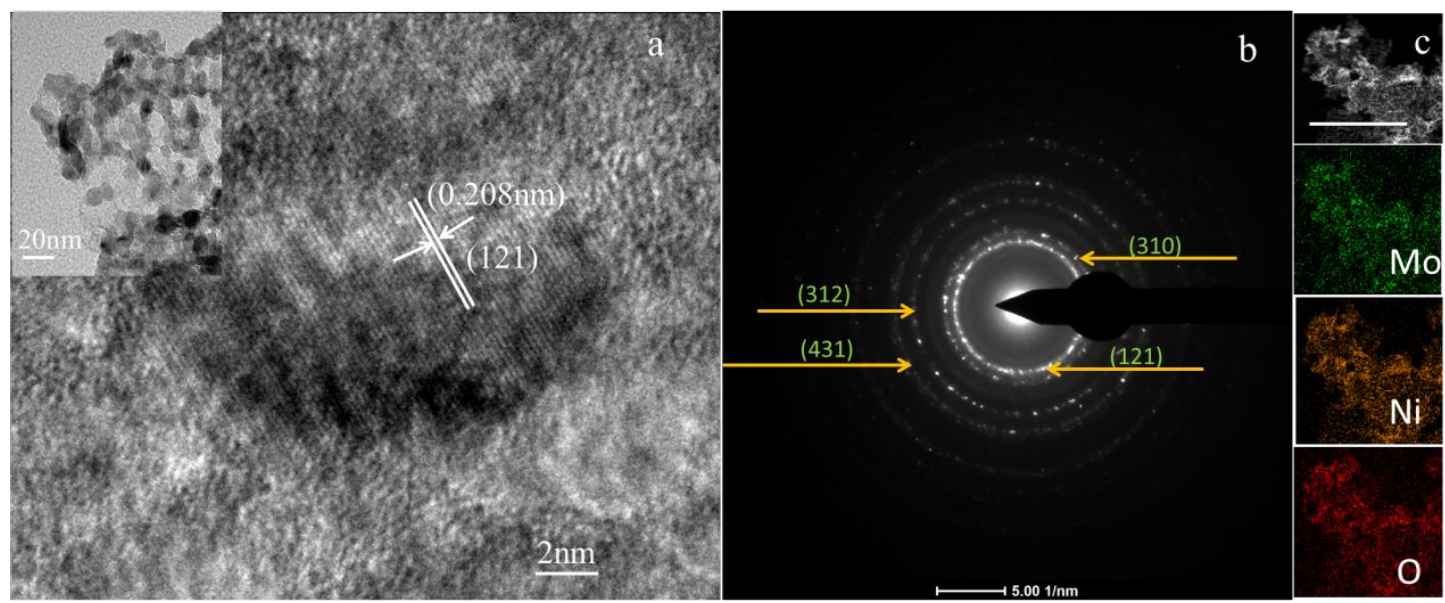

Figure S10. (a) SAED pattern and (b) HRTEM image of the $\mathrm{MoNi}_{4}$ nanoparticles from MoNi-HS-A; (c) Corresponding elemental mapping images of MoNi-HS-A sample. Scale bar, $500 \mathrm{~nm}$. (insert in a is the TEM image of $\mathrm{MoNi}_{4}$ alloys particles )
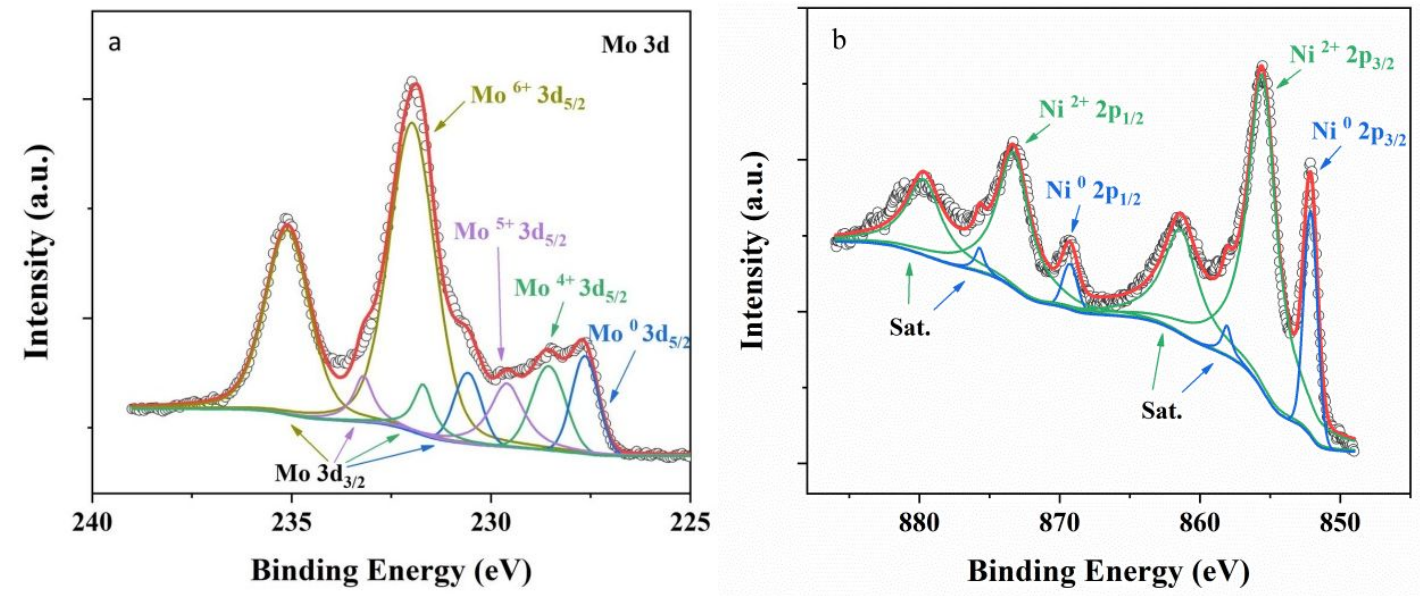

Figure S11. XPS spectra of (a) Mo 3d and (b) Ni 2p of MoNi-HS-A. 


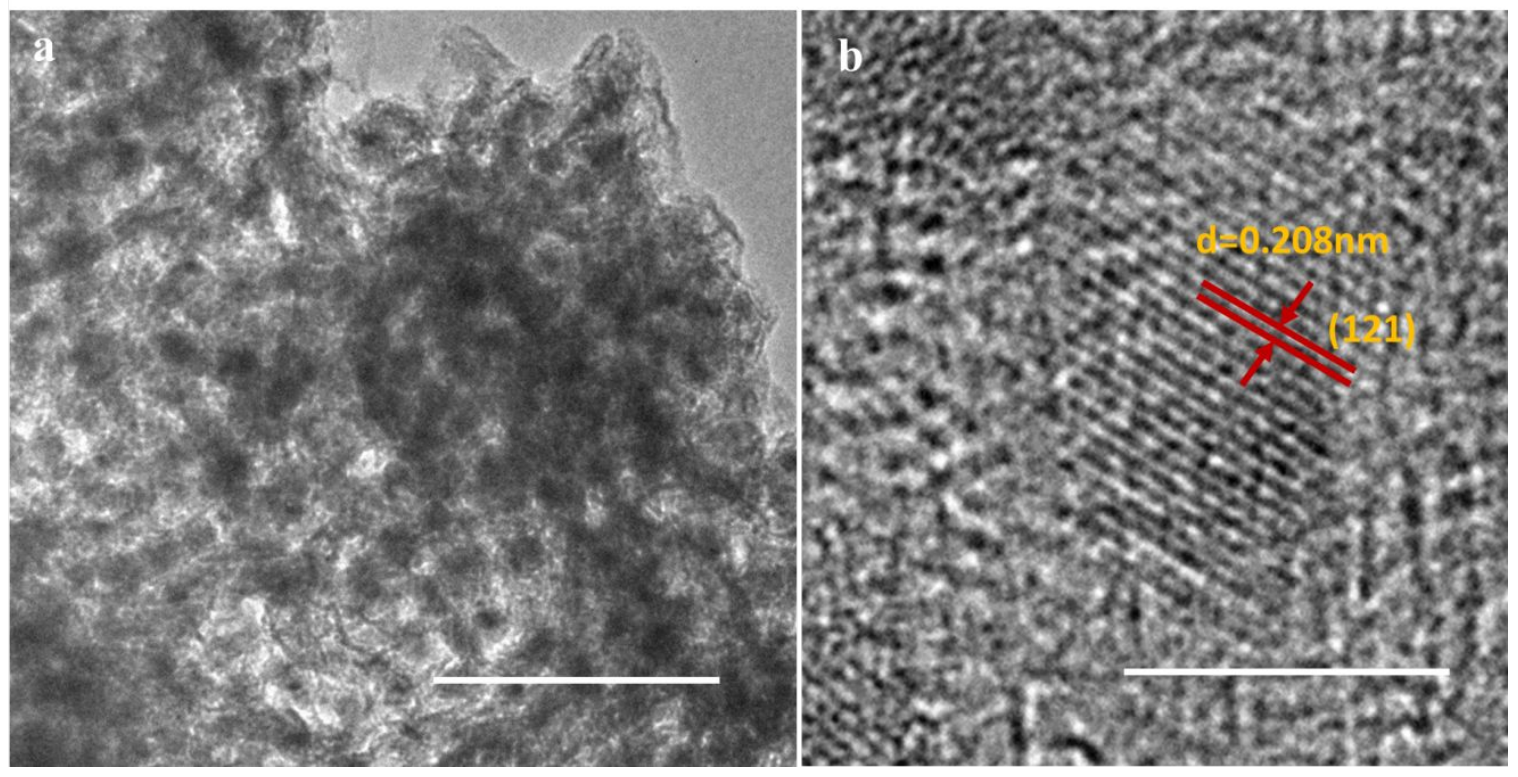

Figure S12. (a)TEM and (b) HRTEM image of MoNi-HS sample after stability test. Scale bars, a: $100 \mathrm{~nm}$; b: $5 \mathrm{~nm}$
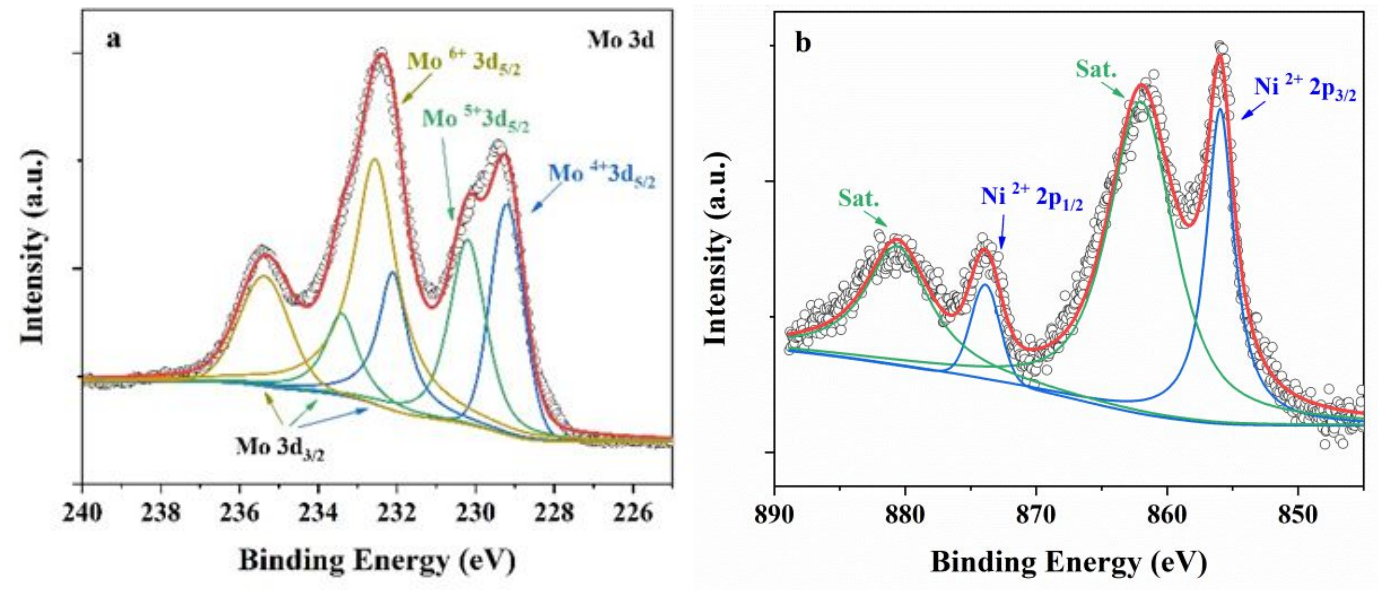

Figure S13. XPS spectra of (a) Mo 3d and (b) Ni 2p of MoNi-HS after stability test. 


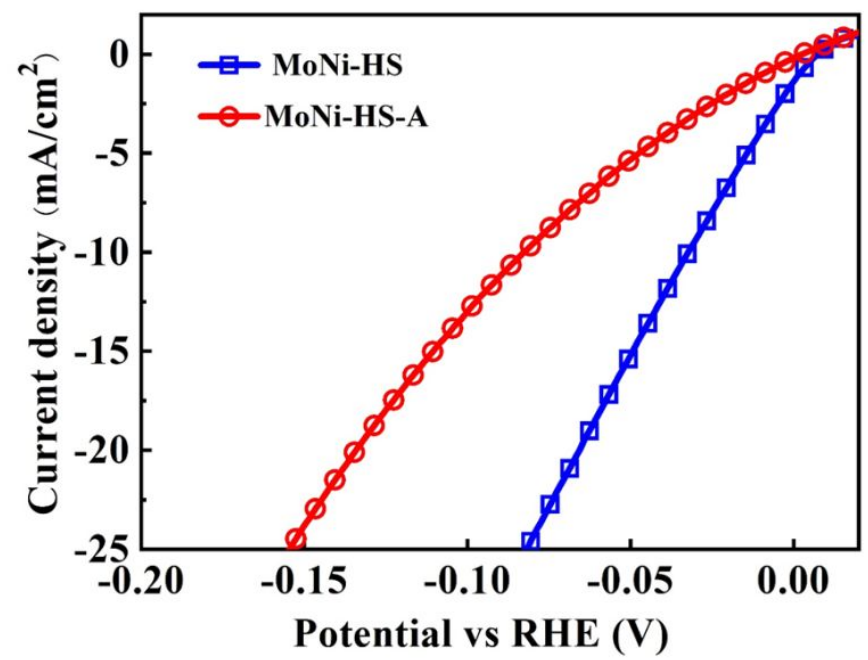

Figure S14. Polarization curves of MoNi-HS and MoNi-HS- A samples in 1M KOH for HER reaction.
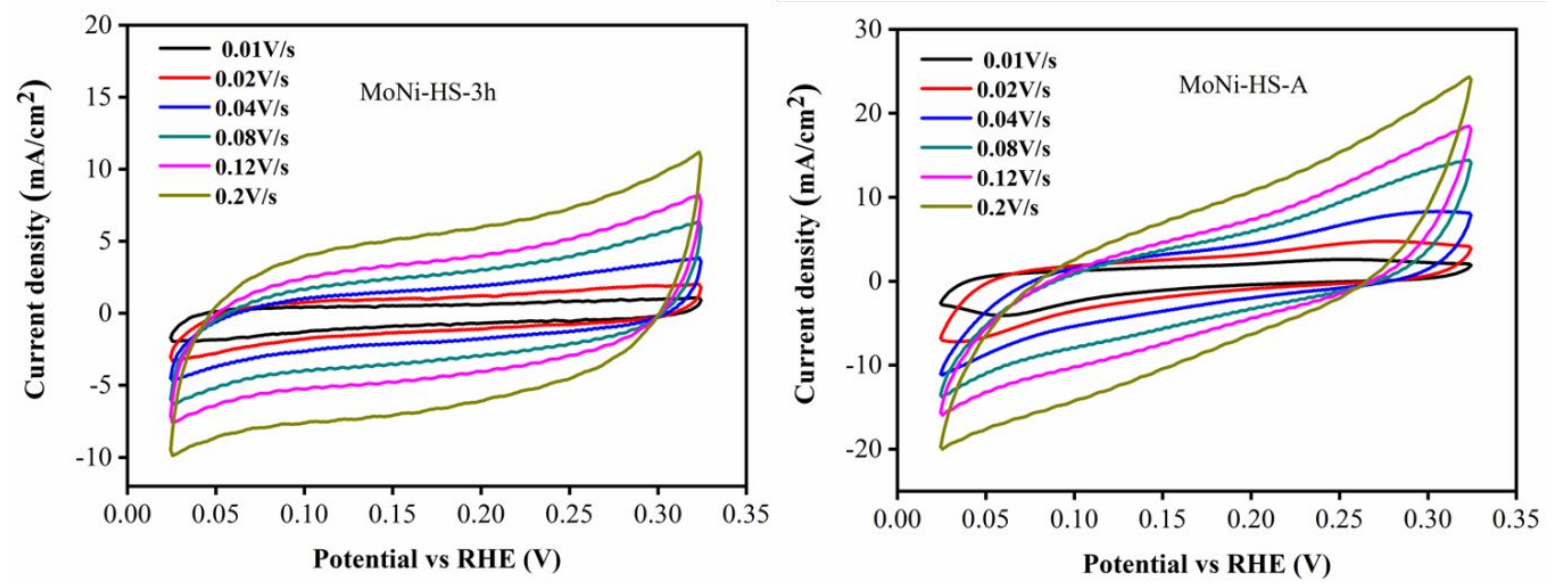

Figure S15.Cyclic voltammograms taken from MoNi-HS and MoNi-HS-A in $1 \mathrm{M} \mathrm{KOH}$ for HER reaction. 


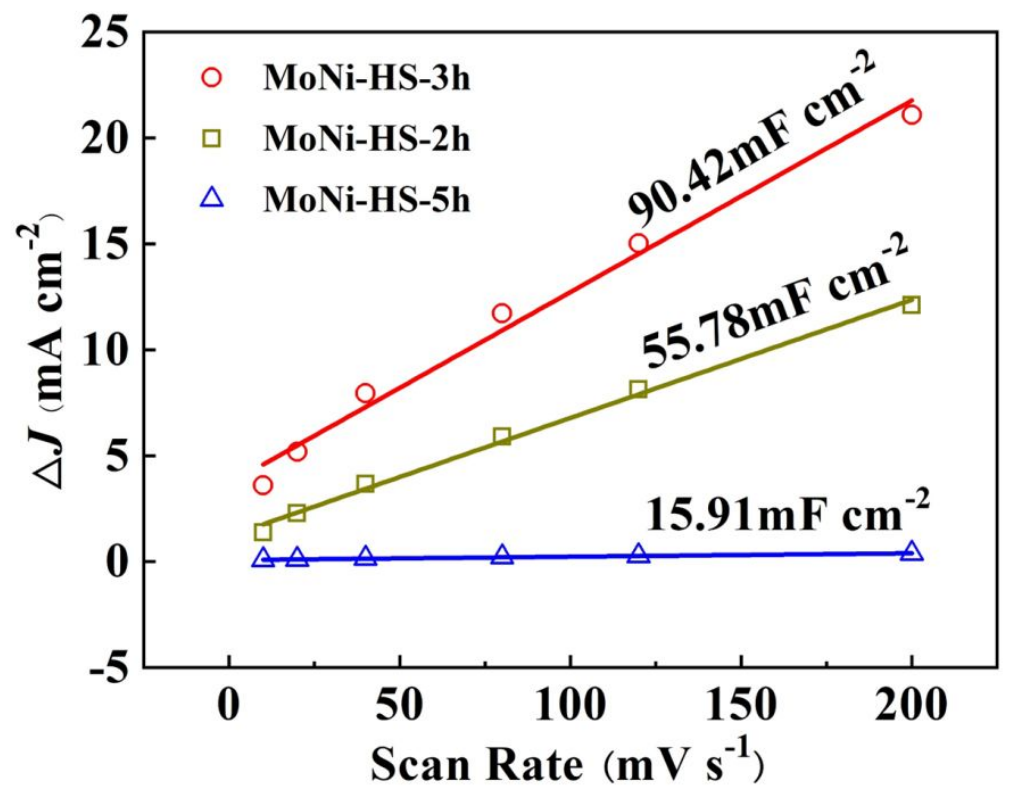

Figure S16.Capacitive J versus scan rate of MoNi-HS with reaction time of $2 \mathrm{~h}$, $3 \mathrm{~h}$ and $5 \mathrm{~h}$.
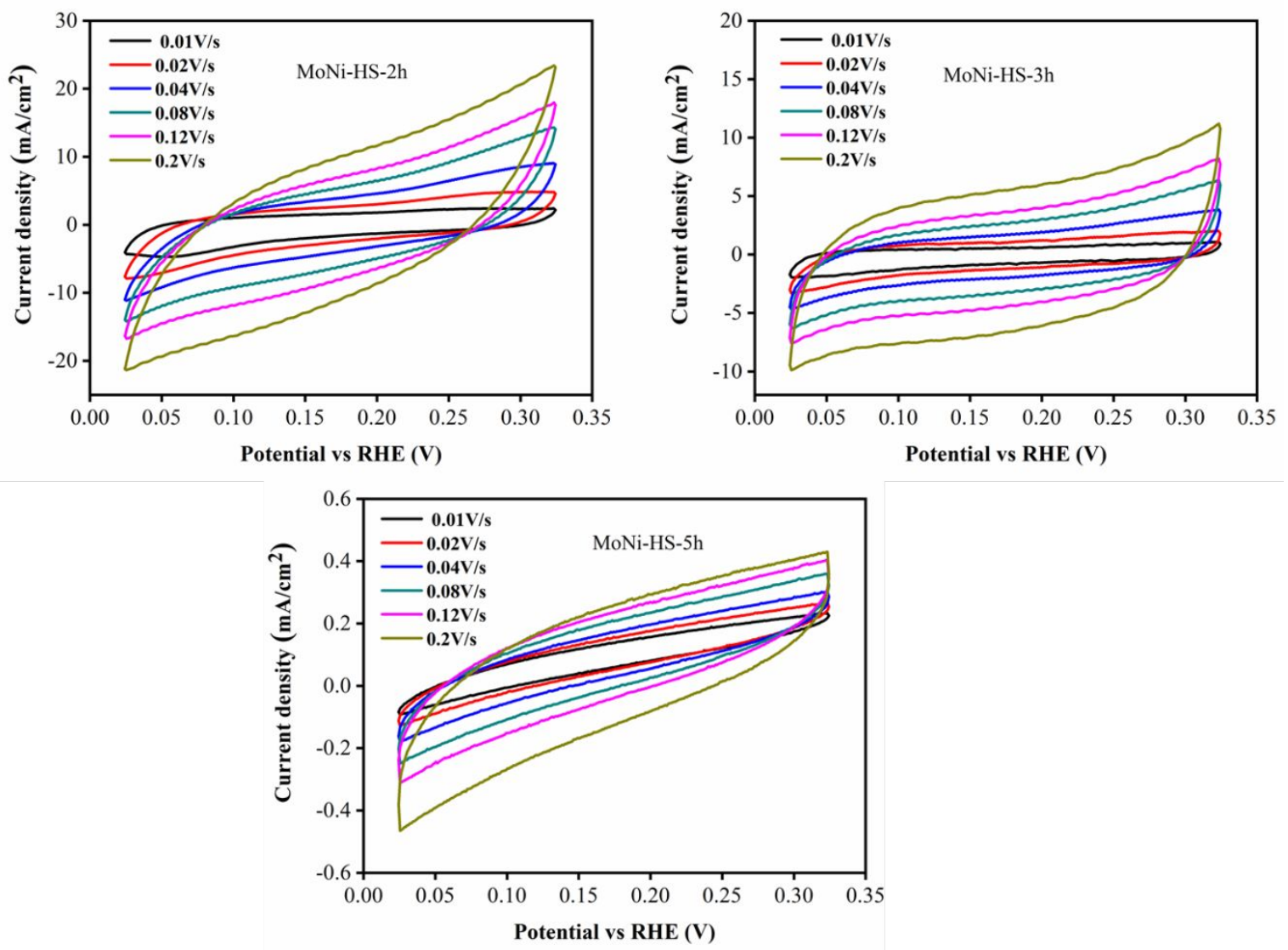

Figure S17.Cyclic voltammograms taken from MoNi-HS with reaction time of $2 \mathrm{~h}, 3 \mathrm{~h}$ and $4 \mathrm{~h}$ in $1 \mathrm{M} \mathrm{KOH}$ for HER reaction. 


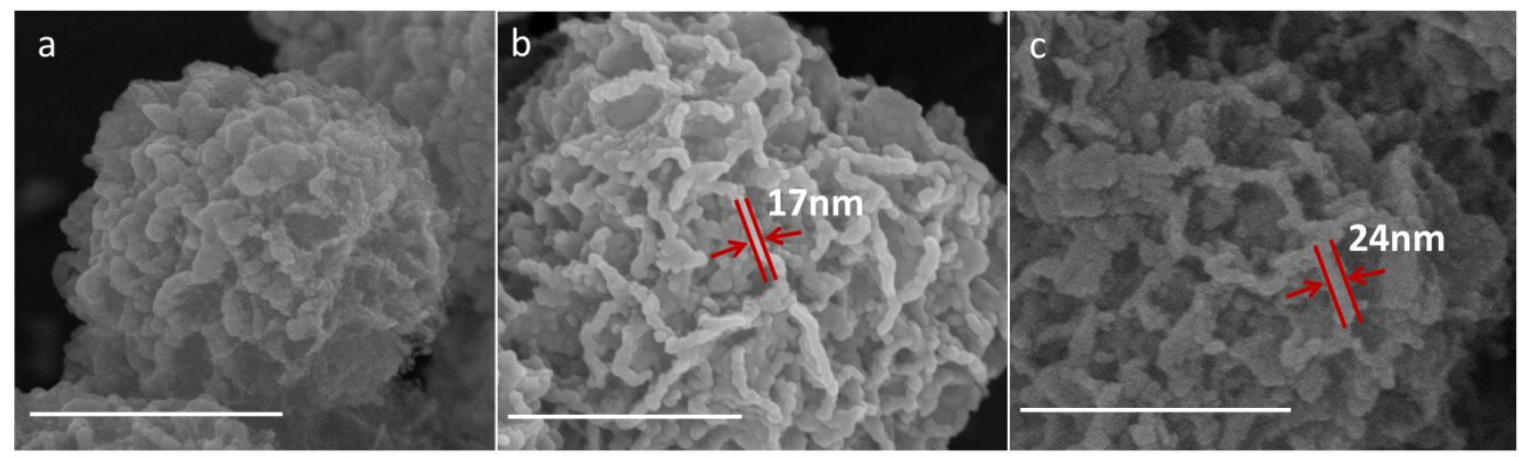

Figure S18.SEM images of MoNi-HS with reaction time of (a) 2h, (b) $3 \mathrm{~h}$ and (c) $5 \mathrm{~h}$. Scale bar: $500 \mathrm{~nm}$.

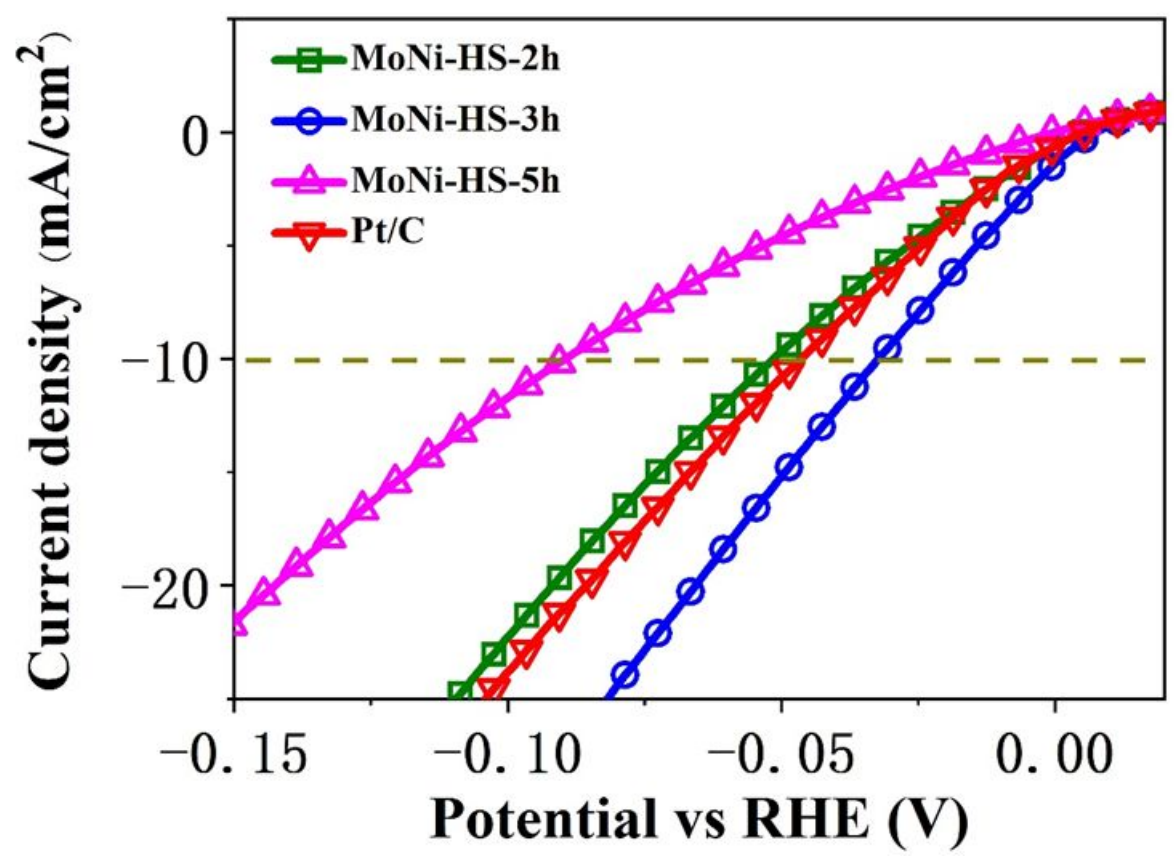

Figure S19. Polarization curves of MoNi-HS with reaction time of $2 \mathrm{~h}, 3 \mathrm{~h}$ and $5 \mathrm{~h}$ in $1 \mathrm{M} \mathrm{KOH}$ for HER. 


\begin{tabular}{|c|c|c|c|c|c|}
\hline Catalysts & Electrolyte & Substrate & $\begin{array}{c}\text { Overpotential } \\
\left(\mathrm{mV} @ 10 \mathrm{~mA} \mathrm{~cm}{ }^{-2}\right)\end{array}$ & $\begin{array}{l}\text { Tafel slope } \\
\left(\mathrm{mV} \mathrm{dec}{ }^{-1}\right)\end{array}$ & Reference \\
\hline $\mathrm{MoNi} 4 / \mathrm{MoO}_{2}$ & $1 \mathrm{M} \mathrm{KOH}$ & Ni Foam & 15 & 30 & 1 \\
\hline $\mathrm{MoNi}_{4} / \mathrm{MoO}_{3-\mathrm{x}}$ & $1 \mathrm{M} \mathrm{KOH}$ & Ni Foam & 17 & 36 & 2 \\
\hline $2 \mathrm{D}-\mathrm{MoS}_{2} / \mathrm{Co}(\mathrm{OH})_{2}$ & $1 \mathrm{M} \mathrm{KOH}$ & $\mathrm{GCE}^{\mathrm{a}}$ & 125 & 76 & 3 \\
\hline $\mathrm{NiMo}_{3} \mathrm{~S}_{4}$ & $0.1 \mathrm{M} \mathrm{KOH}$ & GCE & 275 & 98 & 4 \\
\hline $\mathrm{MoB} / \mathrm{g}-\mathrm{C}_{3} \mathrm{~N}_{4}$ & $1 \mathrm{M} \mathrm{KOH}$ & GCE & 133 & 46 & 5 \\
\hline $\mathrm{MoS}_{2} / \mathrm{Ni}_{3} \mathrm{~S}_{2}$ & $1 \mathrm{M} \mathrm{KOH}$ & Ni Foam & 110 & 83 & 6 \\
\hline $\mathrm{Ni}_{3} \mathrm{~S}_{2} @ \mathrm{MoS}_{2} / \mathrm{FeOOH}$ & $1 \mathrm{M} \mathrm{KOH}$ & Ni Foam & 95 & 85 & 7 \\
\hline $\mathrm{NiS}_{2} / \mathrm{MoS}_{2}$ & $1 \mathrm{M} \mathrm{KOH}$ & GCE & 204 & 65 & 8 \\
\hline $\mathrm{MoP}$ & $1 \mathrm{M} \mathrm{KOH}$ & GCE & 130 & 48 & 9 \\
\hline $\mathrm{MoC}_{\mathrm{x}}$ octahedrons & $1 \mathrm{M} \mathrm{KOH}$ & GCE & 151 & 59 & 10 \\
\hline Cubic-CoSe ${ }_{2}$ & $1 \mathrm{M} \mathrm{KOH}$ & Carbon Cloth & 200 & 85 & 11 \\
\hline $\mathrm{CoP}$ & $1 \mathrm{M} \mathrm{KOH}$ & Carbon Cloth & 240 & 129 & 12 \\
\hline $\mathrm{Ni}_{3} \mathrm{~N} @ \mathrm{CQDS}$ & $1 \mathrm{M} \mathrm{KOH}$ & GCE & 69 & 108 & 13 \\
\hline $\mathrm{Cu} \mathrm{NDS} / \mathrm{Ni}_{3} \mathrm{~S}_{2}$ & $1 \mathrm{M} \mathrm{KOH}$ & Carbon fiber paper & 128 & 76.2 & 14 \\
\hline $\mathrm{MoSe}_{2} @ \mathrm{Ni}_{0.85} \mathrm{Se}$ & $1 \mathrm{M} \mathrm{KOH}$ & GCE & 117 & 66 & 15 \\
\hline $\mathrm{MoS}_{2} / \mathrm{NiOOH}$ & $1 \mathrm{M} \mathrm{KOH}$ & GCE & 73 & 75 & 16 \\
\hline $\mathrm{NiS}_{2} \mathrm{HS}$ & $1 \mathrm{M} \mathrm{KOH}$ & GCE & 219 & 157 & 17 \\
\hline MoNi- HS & $1 \mathrm{M} \mathrm{КОН}$ & GCE & 38 & 31.4 & This work \\
\hline
\end{tabular}

a) GCE: Glassy carbon electrode

Table S1. HER performances of non-noble metal based catalysts in alkaline electrolytes

References.

1. Zhang, J.; Wang, T.; Liu, P.; Liao, Z. Q.; Liu, S. H.; Zhuang, X. D.; Chen, M. W.; Zschech, E.;

Feng, X. L. Efficient Hydrogen Production on $\mathrm{MoNi}_{4}$ Electrocatalysts with Fast Water

Dissociation Kinetics. Natur.Comm. 2017, 8, 15437.

2. Chen, Y. Y.; Zhang, Y.; Zhang, X.; Tang, T.; Luo, H.; Niu, S.; Dai, Z. H.; Wan, L. J.; Hu, J.

S. Self-Templated Fabrication of $\mathrm{MoNi}_{4} / \mathrm{MoO}_{3-\mathrm{x}}$ Nanorod Arrays with Dual Active

Components for Highly Efficient Hydrogen Evolution. Adv. Mater. 2017, 29, 1703311.

3. Zhu, Z. J.; Yin, H. J.; He, C.T.; Al-Mamun, M.; Liu, P. R.; Jiang, L. X.; Zhao, Y.; Wang, Y.;

Yang, H.G.; Tang, Z.Y.; Wang, D.; Chen, X.M.; Zhao, H.J. Ultrathin Transition Metal

Dichalcogenide/3d Metal Hydroxide Hybridized Nanosheets to Enhance Hydrogen Evolution

Activity. Adv. Mater. 2018, 30, 1801171.

4. Jiang, J.; Gao, M. R.; Sheng, W.C.; Yan, Y. S. Hollow Chevrel - Phase $\mathrm{NiMo}_{3} \mathrm{~S}_{4}$ for 
Hydrogen Evolution in Alkaline Electrolytes, Angew. Chem. Int. Ed. 2016, 55, 15240-15245.

5. Zhuang, Z. C.; Li, Y.; Li, Z. L.; Lv, F.; Lang, Z. Q.; Zhao, K. N.; Zhou, L.; Moskaleva, L.; Guo,S.J.; Mai, L. Q. MoB/g - $\mathrm{C}_{3} \mathrm{~N}_{4}$ Interface Materials as a Schottky Catalyst to Boost Hydrogen Evolution, Angew. Chem. Int.Ed. 2018, 57, 496-500.

6. Zhang, J.; Wang, T.; Pohl, D.; Rellinghaus, B.; Dong, R. H.; Liu, S. H.; Zhuang, X.D.; Feng, X.L. Interface engineering of $\mathrm{MoS}_{2} / \mathrm{Ni}_{3} \mathrm{~S}_{2}$ heterostructures for highly enhanced electrochemical overall water splitting activity. Angew. Chem. Int. Ed. 2016, 55, 6702-6707.

7. Zheng, M. Y.; Guo, K. L.; Jiang, W. J.; Tang, T.; Wang, X.Y.; Zhou, P. P.; Du, J.; Zhao, Y. Q.; Xu, C. L.; Hu, J. S. When $\mathrm{MoS}_{2}$ meets FeOOH: A “one-stone-two-birds” heterostructure as a bifunctional electrocatalyst for efficient alkaline water splitting. Appl. Catal.B: Environ. 2019, 244, 1004-1012.

8. Kuang, P. Y.; Tong, T.; Fan, K.; Yu, J.G. In situ Fabrication of Ni-Mo bimetal Sulfide Hybrid as an Efficient Electrocatalyst for Hydrogen Evolution over Wide pH Range. ACS Catal. 2017, 7, 6179-6187.

9. Xiao, P.; Alam Sk, M.; Thia, L.; Ge, X.M.; Lim, R. J.; Wang, J.Y.; Lim, K.H.; Wang, X. Molybdenum Phosphide as an Efficient Electrocatalyst for The Hydrogen Evolution Reaction. Energy Environ. Sci. 2014, 7, 2624-2629.

10. Wu, H.B.; Xia, B.Y.; Yu, L.; Yu, X.Y.; Lou, X.W. Porous Molybdenum Carbide Nano-Octahedrons Synthesized via Confined Carburization in Metal-Organic Frameworks for Efficient Hydrogen Production. Nat. Commun. 2015, 6, 6512-6519.

11. Chen, P. Z.; Xu. K.; Tao, S.; Zhou, T. P.; Tong, Y.; Ding, H.; Zhang, L. D.; Chu, W. S.; Xie, Y. Phase - Transformation Engineering in Cobalt Diselenide Realizing Enhanced Catalytic Activity for Hydrogen Evolution in an Alkaline Medium. Adv. Mater. 2016, 28, 7527-7532.

12. Tian, J. Q.; Liu, Q.; Asiri, A. M.; Sun, X. P. Self-Supported Nanoporous Cobalt Phosphide Nanowire Arrays: an Efficient 3D Hydrogen-Evolving Cathode over the Wide Range of pH 0-14. J. Am.Chem. Soc. 2014,136, 7587-7590.

13. Zhou, M.; Weng, Q.H.; Popov, Z.I.; Yang, Y.J.; Antipina, L.Y.; Sorokin, P. B.; Wang, X.; 
Bando, Y.; Golberg, D. Construction of Polarized Carbon-Nickel Catalytic Surfaces for Potent, Durable, and Economic Hydrogen Evolution Reactions. ACS Nano 2018, 12, 41484155.

14. Feng, J. X.; Wu, J.Q.; Tong, Y. X.; Li, G.R. Efficient Hydrogen Evolution on Cu Nanodots-Decorated $\mathrm{Ni}_{3} \mathrm{~S}_{2}$ Nanotubes by Optimizing Atomic Hydrogen Adsorption and Desorption. J. Am. Chem. Soc. 2018,140, 610-617.

15. Wang, C. Q.; Zhang, P.; Lei, J. L.; Dong, W.; Wang, J. H.; Integrated 3d MoSe ${ }_{2} @ \mathrm{Ni}_{0.85} \mathrm{Se}$ Nanowire Network with Synergistic Cooperation as Highly Efficient Electrocatalysts for Hydrogen Evolution Reaction in Alkaline Medium. Electrochim. Acta 2017,246, 712-719.

16. Zhang, X.; Liang, Y. Y. Nickel Hydr(oxy)oxide Nanoparticles on Metallic $\mathrm{MoS}_{2}$ Nanosheets: a Synergistic Electrocatalyst for Hydrogen Evolution Reaction. Adv. Sci.2018, 5, 1700644.

17. Tian, T.; Huang, L.; Ai, L.H.; Jiang, J. Surface Anion-Rich NiS $\mathrm{S}_{2}$ Hollow Microspheres Derived from Metal-Organic Frameworks as a Robust Electrocatalyst for the Hydrogen Evolution Reaction, J. Mater. Chem. A, 2017, 5, 20985-20992. 